\title{
Article \\ Ab Initio Spectroscopic Investigation of Pharmacologically Relevant Chiral Molecules: The Cases of Avibactam, Cephems, and Idelalisib as Benchmarks for Antibiotics and Anticancer Drugs
}

\author{
Elena Molteni $^{1,2, *(\mathbb{D})}$, Giovanni Onida ${ }^{1,2} \mathbb{D}$, Matteo Ceccarelli ${ }^{3,4}\left(\mathbb{D}\right.$ and Giancarlo Cappellini ${ }^{2,3,4}(\mathbb{D}$ \\ 1 Department of Physics, Università degli Studi di Milano, via Celoria 16, 20133 Milano, Italy; \\ giovanni.onida@mi.infn.it \\ 2 European Theoretical Spectroscopy Facility (ETSF, www.etsf.eu) \\ 3 Department of Physics, Università degli Studi di Cagliari, Cittadella Universitaria di Monserrato, \\ S.P. Monserrato-Sestu Km. 0.700, 09042 Monserrato, Italy; matteo.ceccarelli@dsf.unica.it (M.C.); \\ giancarlo.cappellini@dsf.unica.it (G.C.) \\ 4 CNR-IOM SLACS Cagliari, Università degli Studi di Cagliari, Cittadella Universitaria di Monserrato, \\ S.P. Monserrato-Sestu Km. 0.700, 09042 Monserrato, Italy \\ * Correspondence: elena.molteni@mi.infn.it
}

\section{check for}

updates

Citation: Molteni, E.; Onida, G.; Ceccarelli, M.; Cappellini, G. Ab Initio Spectroscopic Investigation of Pharmacologically Relevant Chiral Molecules: The Cases of Avibactam, Cephems and Idelalisib as Benchmarks for Antibiotics and Anticancer Drugs. Symmetry 2021, 13, 601. https://doi.org/10.3390/ sym13040601

Academic Editor: Tomohiro Inagaki

Received: 5 March 2021

Accepted: 28 March 2021

Published: 3 April 2021

Publisher's Note: MDPI stays neutral with regard to jurisdictional claims in published maps and institutional affiliations.

Copyright: (c) 2021 by the authors. Licensee MDPI, Basel, Switzerland. This article is an open access article distributed under the terms and conditions of the Creative Commons Attribution (CC BY) license (https:/ / creativecommons.org/licenses/by/ $4.0 /)$.

\begin{abstract}
The ability to accurately measure or predict several physicochemical properties of molecules which play a role as active substances in drugs can be of strategic importance for pharmacological applications, in addition to its possible interest in fundamental research. Chirality is a relevant feature in the characterization of drug molecules: enantiomers can show different pharmacological activity and adverse effects. The ability to separate stereoisomers and to assign their absolute configuration can thus be crucial. Circular dichroism (CD) spectra are a useful tool to distinguish between enantiomers. In this work we apply an in-house developed code, based on an efficient DFT approach for circular dichroism, to fully characterize the molecular optical properties in the case of few selected fundamental molecules for current medical and pharmaceutical research, namely avibactam, as representative of non $\beta$-lactam inhibitors, two cephems (cefepime and cefoxitin), as examples of $\beta$-lactam antibiotics, and idelalisib, as a recent relevant anticancer active substance to treat major leukemias. For the above molecules, in addition to their optical absorption spectra, we calculate their CD spectra within state-of-the-art computational techniques. We then investigate both the conformational and chemical sensitivity of absorption and CD spectra for the chosen molecules. The outcomes of the present research could be of fundamental importance to gain additional information on molecules involved in therapeutic protocols for severe diseases or in drug design.
\end{abstract}

Keywords: circular dichroism; molecular chirality; ab initio calculation

\section{Introduction}

Studies on molecules which play a role in medicine and pharmacology are nowadays of strategic importance both for fundamental research and for the direct impact on drug design and on therapeutic protocols.

Chronic lymphocytic leukemia (CLL) could be considered as the most common form of leukemia in the western world. CLL is a progressive hematologic disease characterized by an increase of the number of monoclonal mature B cells present in the blood, in the bone marrow and secondary lymphatic elements of the patients [1]. The use of drugs containing the idelalisib molecule as active substance has recently been successfully applied in healing protocols for CLL and other leukemias, especially for patients who are less able to undergo chemotherapy [1]. Idelalisib inhibits PI3K (phosphatidylinositol 3-kinase p110) a compound which is hyperactive in B cell malignancies. 
Another fundamental issue in drug design is the problem of antibacterial resistance, especially for Gram negative species where a permeation issue exists [2]. For many decades $\beta$-lactam compounds have been the most important drugs to attack bacterial infections [3]. Resistance to the $\beta$-lactam antibiotics is a major and growing problem [3]. One of the most important mechanisms against $\beta$-lactam antibacterials in Gram-negative bacteria involves $\beta$-lactamases which catalyze the hydrolysis of the $\beta$-lactam ring [3], making molecules no longer active on the target. In recent years the avibactam molecule [3,4], which is not itself a $\beta$-lactam, has been demonstrated to be a wide-spectrum serine $\beta$ lactamase inhibitor; avibactam has a core ring structure which enables inhibition via a mechanism involving covalent modification of a nucleophilic serine residue and its binding is reversible [5]. Several combinations of $\beta$-lactam antibiotics and $\beta$-lactamase inhibitors have been investigated [3] in order to overcome bacterial resistance to $\beta$-lactam antibiotics. In particular, avibactam in combination with ceftazidime has been reported as a valid therapy for patients with ceftazidime-resistant Enterobacteriaceae and P. aeruginosa infections [6]. Avibactam is the first of the clinically useful nucleophilic serine enzyme inhibitors that contain a diazabicyclooctane (DBO) core [7]. Its inhibitory properties have been also measured across a large variety of enzymes considered for their clinical importance [8]. Through chemical modifications we have today diverse diazabicyclooctane derivatives [9].

Cephems are a subgroup of $\beta$-lactam antibiotics including cephalosporins and cephamycins. Cefepime is a fourth-generation cephalosporin [10], with a rather extended spectrum of activity against both Gram-positive and Gram-negative bacteria. It exerts its antimicrobial effect by attaching to specific penicillin-binding proteins involved in assembling the bacterial cell wall. Cefoxitin [11] was the first semisynthetic cephamycin to be made available for clinical use. It has a broader spectrum of activity compared to cephalosporins, due to its almost complete resistance to a wide range of beta-lactamases.

Chirality , a property related to the lack of planes of symmetry and inversion centers, plays an important role in molecular recognition; therefore, it is of interest for pharmaceutical science [12], catalysis in chemical industry, and biomolecular function. One enantiomer (i.e., either of two nonsuperimposable mirror images of a chiral drug molecule) may indeed correctly bind in the target binding site, yielding the desired pharmacologic effect, while the other enantiomer may not fit into the binding site, being thus inactive, or it may even interact with a different site causing adverse effects (e.g., [12] and refs therein). The ability to distinguish between enantiomers of a given chiral molecule can therefore be crucial in drug design and production. In specific classes of molecules, such as kinase inhibitors, chiral drug discovery has attracted attention as a method to improve drug molecules, e.g., in terms of solubility or pharmacokinetic profiles [12]. In the case of idelalisib, its chiral character has been reported as contributing to its selectivity towards the PI3K $\delta$ kinase [12].

Chiral molecules display distinguishable enantiomeric response to left- and righthand circularly polarized light (CPL), resulting in optical rotatory dispersion (ORD) and electronic circular dichroism (ECD). Circular dichroism spectroscopy is a very useful experimental technique, showing chemical and conformational [13] sensitivity in addition to allowing the assignment of the absolute configuration. Within the field of pharmaceutically relevant molecules, CD spectroscopy has been shown to be an accurate and precise method for identifying different cephalosporins in common clinical use [14].

However, experimentally measured CD spectra can only be used to assign the absolute configuration of molecules if the chiroptical spectrum of an enantiomer is known. There is indeed no obvious and general correlation between the absolute configuration of a molecule and the sign of its (main) CD peak(s). Empirical rules have only been established within specific classes of molecules [15]. Moreover, for flexible molecules, conformational effects may lead to dramatic changes in circular dichroism spectra, up to reversing the sign of the main CD peaks for a fixed enantiomer [13]. 
Computational studies can yield valuable insight on several properties of drug molecules, allowing a complete control on molecule conformation, which may be difficult or even impossible experimentally. Classical molecular dynamics (MD) simulations, together with Monte Carlo methods, have been applied $[16,17]$ to shed light on the mechanism of $\beta$-lactam antibiotic translocation through bacterial membranes. At a difference with classical MD, ab initio techniques allow the explicit treatment of the system's electronic properties and their effect on spectroscopic features. Within this framework, the optical properties of several both biologically and technologically important molecules, and their dependence on conformational details, chemical substitutions, solvation, and adsorption on surfaces were investigated by some of us in recent years [13,18-26].

In particular, focusing on circular dichroism, by calculating the $C D$ spectra of a fixed molecular enantiomer in its low energy conformations, one can identify the conserved features (if any) and their position in wavelength. This indicates which spectral regions in experimentally measured CD spectra of the molecule can be used to unambiguously assign the absolute configuration (enantiomer) and which regions are instead useful to identify the conformer(s) present in the experimental sample.

Studying the chiroptical properties of molecules can therefore complete the scenario of their electronic and optical properties, yielding information which in some cases may not be collected through other methods.

In this work we characterize the circular dichroism spectra (in addition to optical absorption spectra) of chosen molecules of medical/pharmaceutical interest, by applying an efficient computational approach based on plane-waves density functional theory (DFT), previously developed by some of us [13].

We selected avibactam as an example of a non $\beta$-lactam inhibitor, the two cephems, namely cefepime and cefoxitin, as examples of antibiotic molecules and idelalisb as a recent relevant anticancer active substance for the treatment of major leukemias.

The present results could be useful in the research, design, and production of devices based on optical techniques to recognize and identify molecules of importance in drug design scenarios, such as the quantification of accumulation in bacterial cells $[27,28]$ and may be beneficial to other studies involving the considered molecules and their use in therapeutic protocols for severe syndromes.

\section{Results and Discussion}

In Figure 1 we report the structural formula of the investigated molecules: avibactam, idelalisib, cefepime, and cefoxitin, and in Figure 2 we show the chosen geometries: four different conformers for the avibactam molecule (top panel), two conformers for the idelalisib molecule (left part of bottom panel) and one conformer each for the two chosen cephems, cefepime and cefoxitin (right part of bottom panel). The avibactam structures were taken from: Protein Data Bank (PDB) 4WM9 entry (Acinetobacter Baumanii OXA24 complex with avibactam [29]), PubChem CID 9835049 (avibactam free acid), PDB 4ZAM (Crystal structure of SHV-1 beta-lactamase bound to avibactam [30]), PDB 4ZBE (Crystal structure of KPC-2 beta-lactamase complexed with avibactam [30]). The idelalisib structures were taken from: PubChem CID 11625818, PDB 4XE0 (Idelalisib bound to the P110 subunit of PI3K Delta [31]). The cefepime and cefoxitin structures were taken from the Antimicrobial Compounds Database maintained by G. Malloci (https:/ / www.dsf.unica.it/ translocation/db/, accessed on 4 August 2017 [32]).

We report here some nonexhaustive details of the three types of molecules under study, in the here chosen chemical structures: avibactam, idelalisib, cephems. Avibactam is composed of 28 to 30 atoms, depending on its protonation state: the DFT Kohn-Sham HOMO and LUMO levels of its $4 \mathrm{WM} 9$ structure are at $-5.33 \mathrm{eV}$ and $-2.69 \mathrm{eV}$ respectively, with a gap of $2.64 \mathrm{eV}$. Idelalisib has 49 atoms: for its $4 \mathrm{XE} 0$ structure the HOMO is at -5.97 $\mathrm{eV}$, the LUMO at $-2.55 \mathrm{eV}$, with a gap of $3.42 \mathrm{eV}$. The two cephems, cefepime ad cefoxitin, are composed of 56 and 44 atoms respectively: for cefepime, the $\mathrm{HOMO}$ is at $-4.53 \mathrm{eV}$, the LUMO at $-2.49 \mathrm{eV}$, with a gap of $2.04 \mathrm{eV}$. All the above-mentioned levels are obtained 
with the vacuum level being set at zero energy. As for their chemical composition, all these molecules contain carbon, nitrogen, oxygen, and hydrogen atoms. In addition, avibactam and the two cephems also contain sulfur ( 1 or 2 sulfur atom per molecule in avibactam and cephems respectively), while idelalisib contains one fluorine atom per molecule.
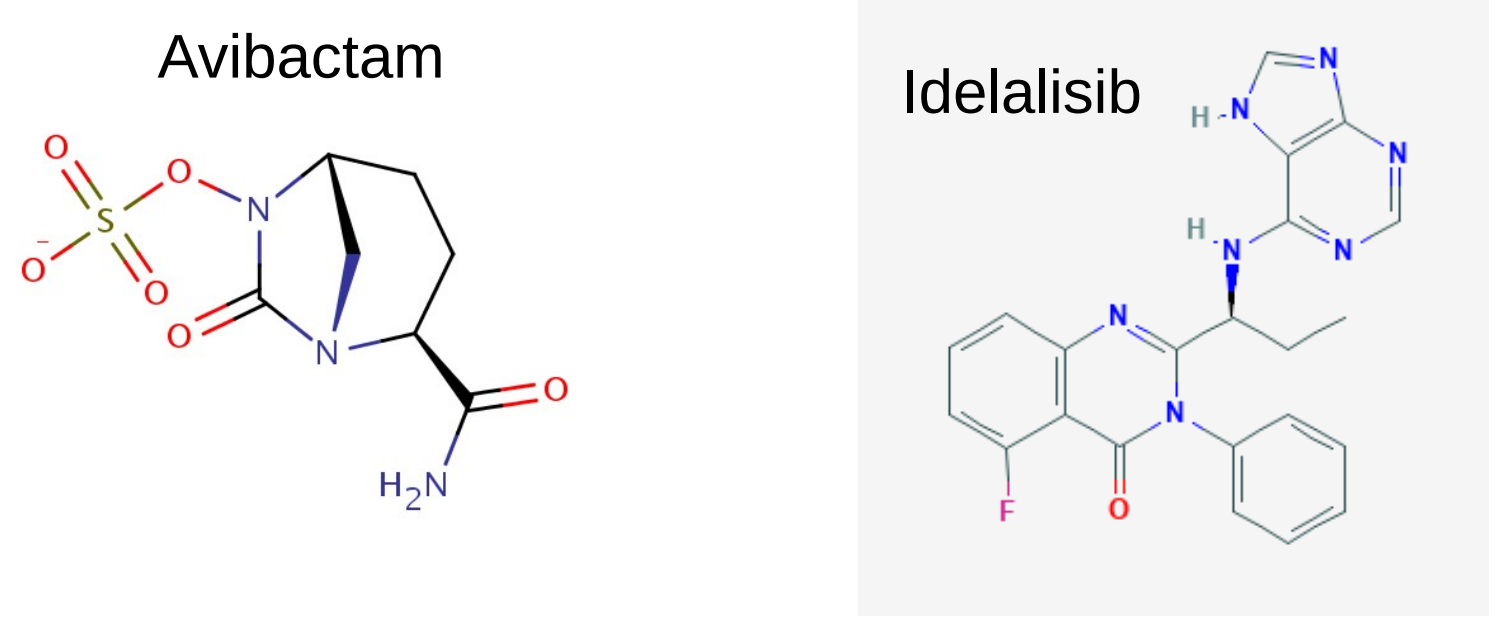

\section{Cefepime}

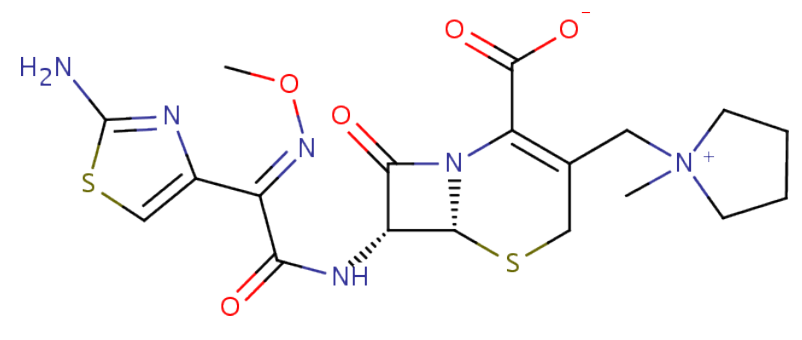

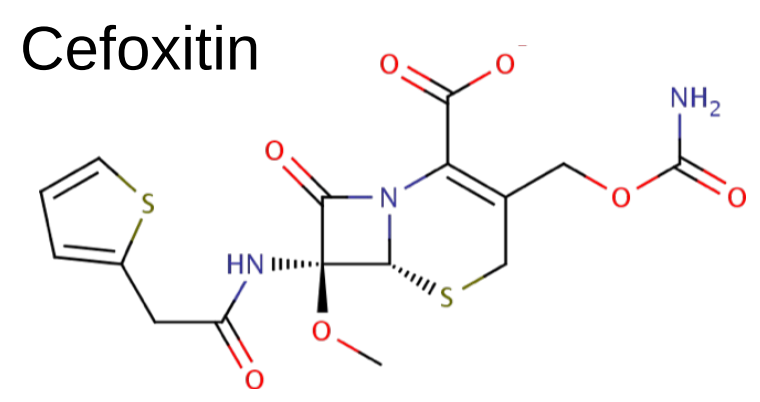

Figure 1. Structural formulas for the investigated molecules: avibactam, idelalisib, cefepime, cefoxitin.

In Figures 3-5 we report the absorption and circular dichroism spectra calculated for the investigated molecules, i.e., avibactam (Figure 3), idelalisib (Figure 4), and the two cephems cefepime and cefoxitin (Figure 5). The choice of four and two conformers for avibactam and idelalisib, respectively, allows us to gain insight on the conformational sensitivity of absorption and CD spectra, while the choice of two different cephems can provide information on the chemical sensitivity of the above-mentioned spectra (Figure 2). 

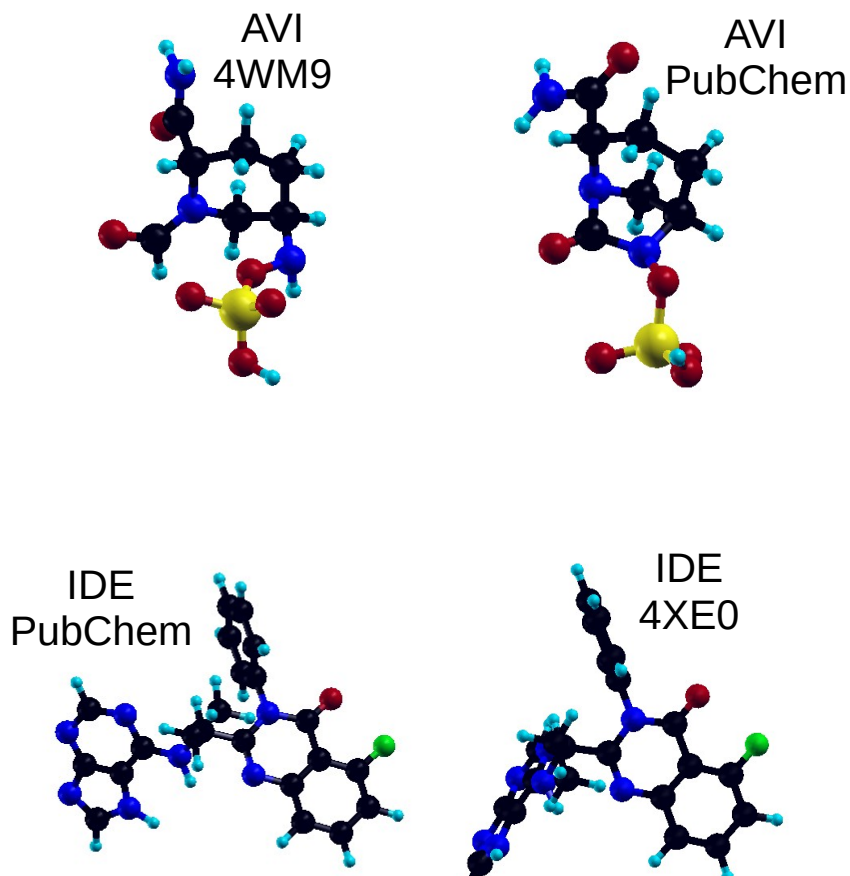

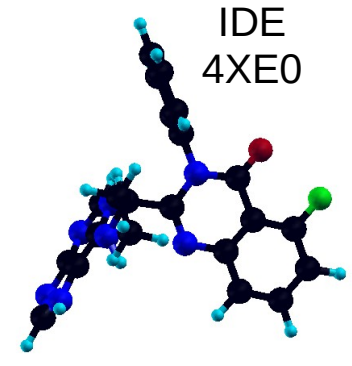

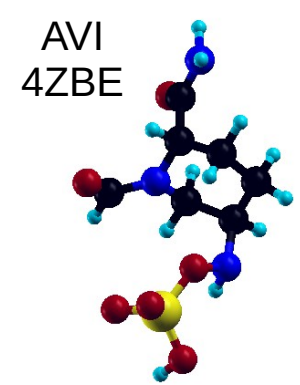
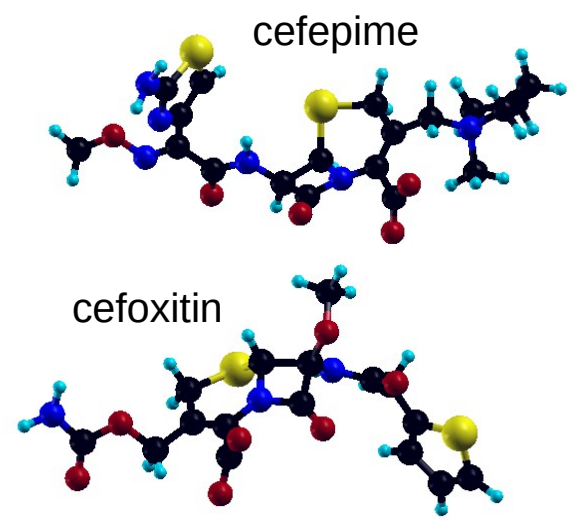

Figure 2. Chemical structure and geometry of the investigated molecules/conformations. Atom color codes: black = carbon, red $=$ oxygen, blue $=$ nitrogen, yellow $=$ sulfur, green $=$ fluorine, light blue $=$ hydrogen .

(a)

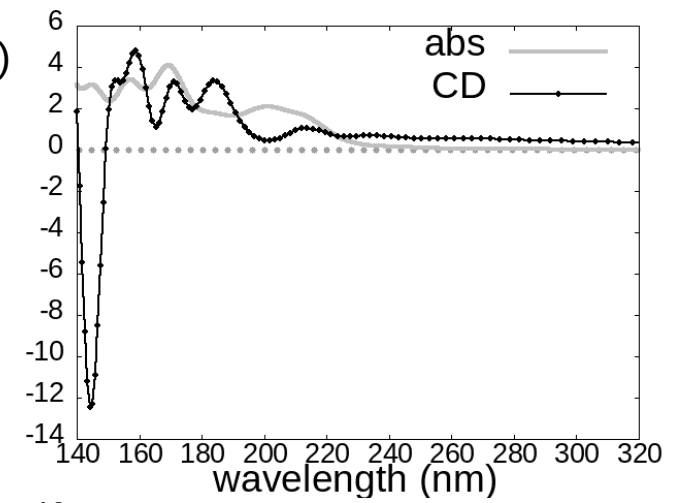

(c)

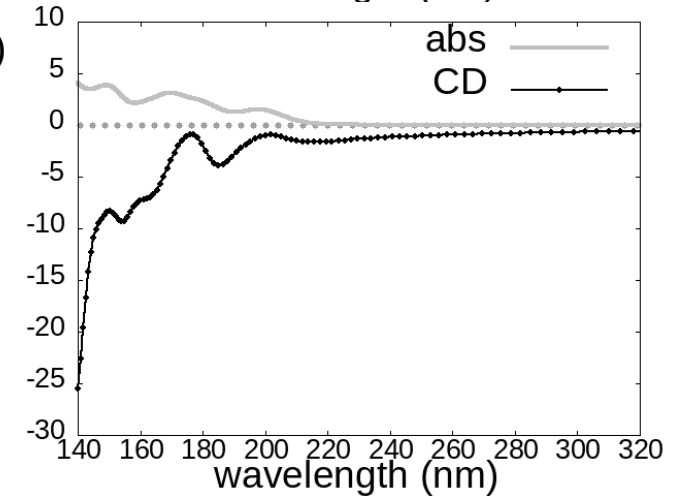

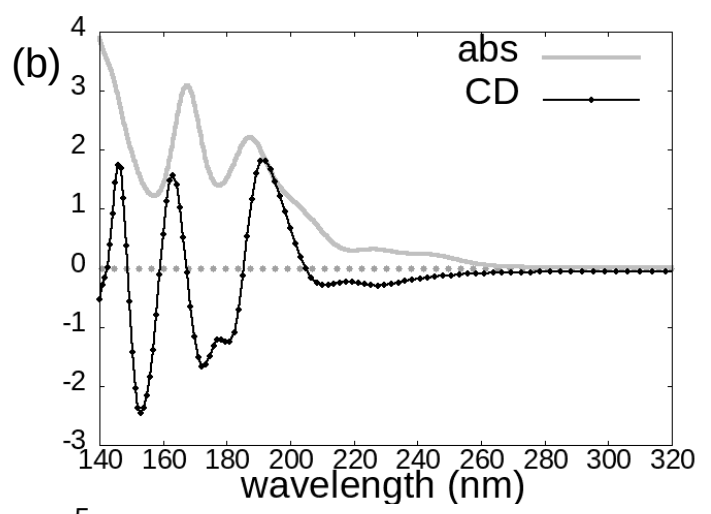

(d)

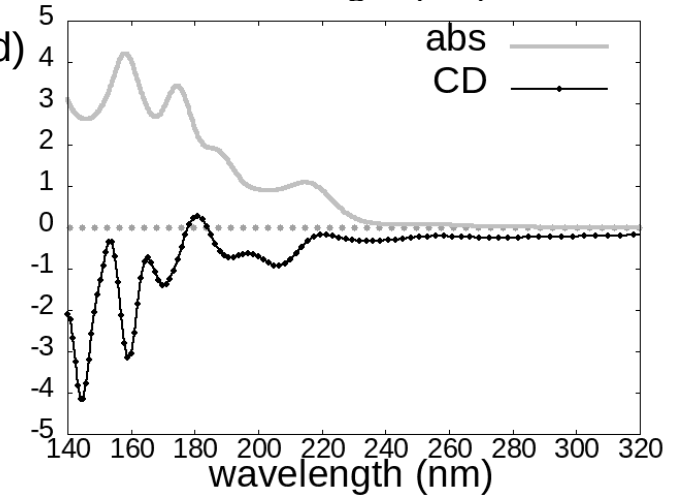

Figure 3. Photoabsorption cross-sections (grey line) and circular dichroism spectra (black line with dots) of the avibactam molecule in the following conformations: (a) 4WM9, (b) PubChem, (c) 4ZAM, (d) 4ZBE. 

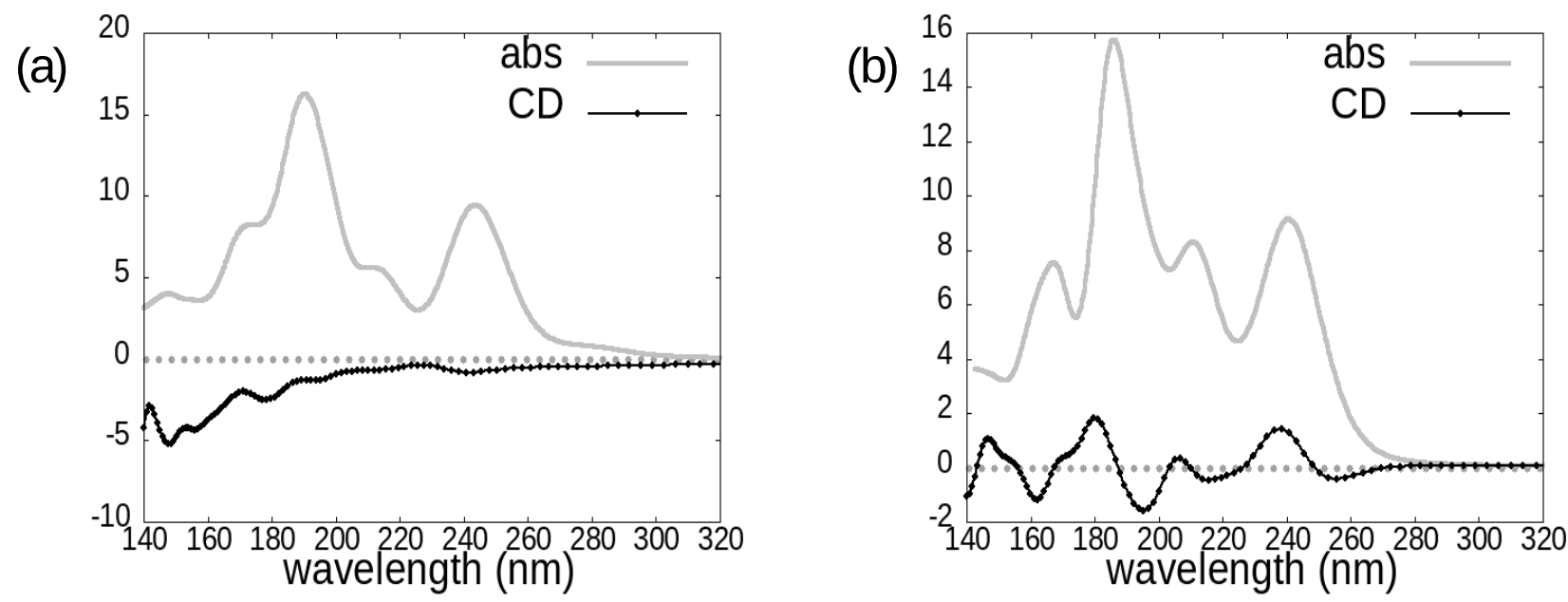

Figure 4. Photoabsorption cross-sections (grey line) and circular dichroism spectra (black line with dots) of the idelalisib molecule in the following conformations: (a) PubChem, (b) 4XE0.
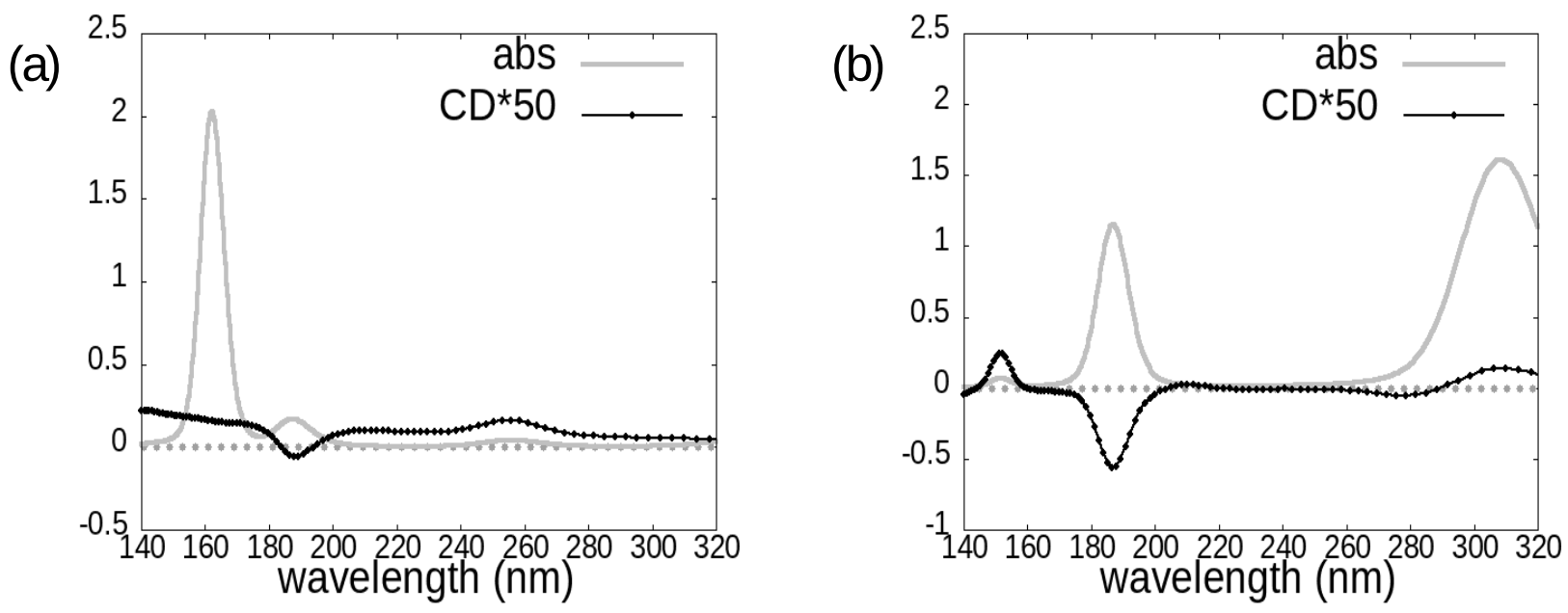

Figure 5. Photoabsorption cross-sections (grey line) and circular dichroism spectra (black line with dots) of the two investigated cephems: (a) cefepime, (b) cefoxitin.

The onset of idelalisib spectra is at around $280 \mathrm{~nm}$; in the case of avibactam it is at around $260 \mathrm{~nm}$ in absorption, while the main CD features appear below $220 \mathrm{~nm}$; as for cephems, computed spectra have in general non-negligible features across the whole analyzed wavelength range, except for cefepime absorption spectrum, which has the most intense absorption features at wavelengths shorter than $200 \mathrm{~nm}$.

We directly compare the spectra of different conformers in the following figures: Figures $6 a, c$ and 7a,b. While in the case of avibactam no clear trend appears (except perhaps a stronger CD negative peak in the 140-160 $\mathrm{nm}$ region displayed by the 4ZAM conformer, see Figure $6 \mathrm{a}, \mathrm{c})$, the case of the idelalisib molecule is exemplar of a strong conformational sensitivity of CD spectra (Figure 7b), despite absorption spectra being extremely similar (Figure 7a). 

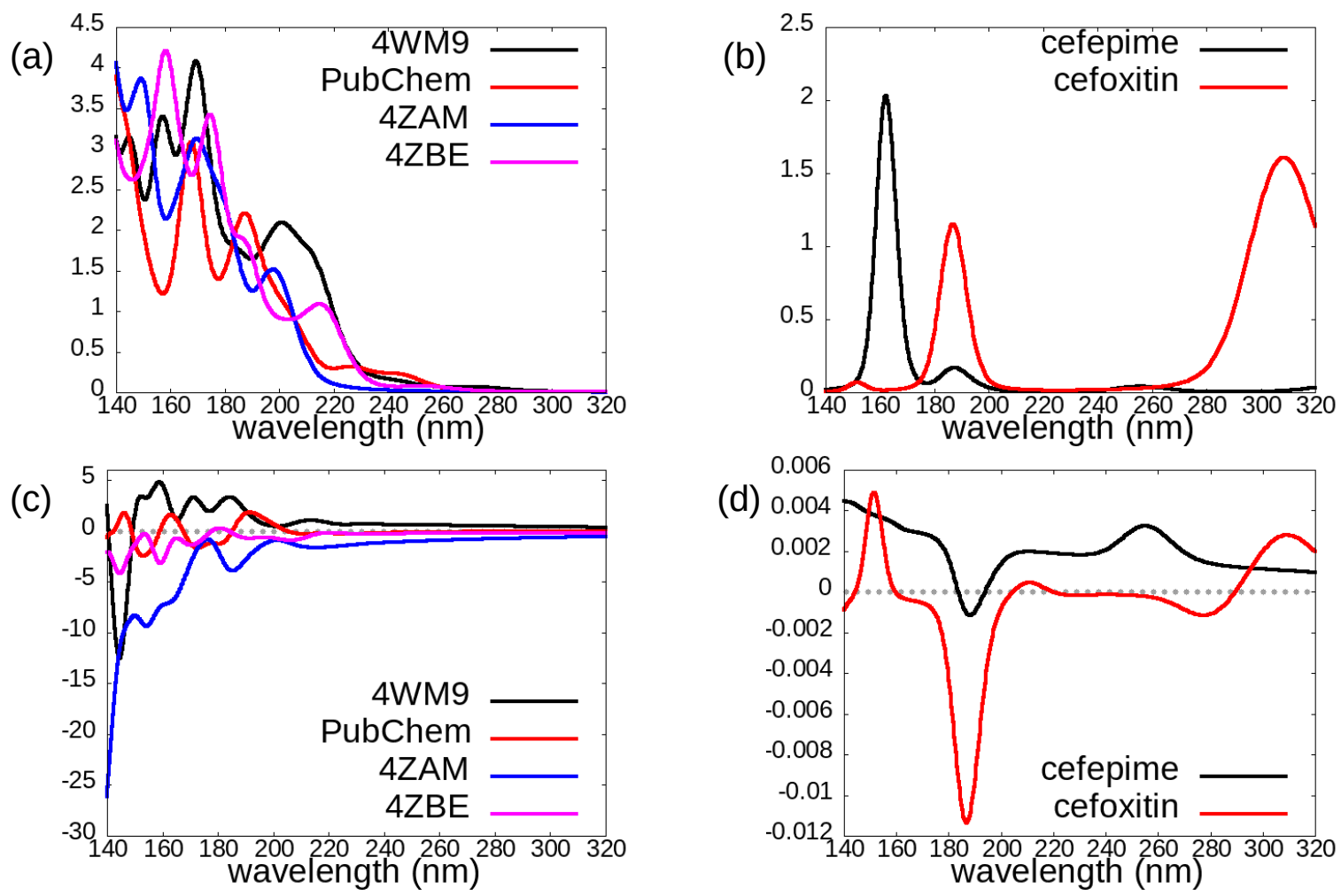

Figure 6. Comparisons of photoabsorption cross sections $(\mathbf{a}, \mathbf{b})$ and circular dichroism $(\mathbf{c}, \mathbf{d})$ for: The four investigated conformations of avibactam $(\mathbf{a}, \mathbf{c})$, and the two investigated cephems $(\mathbf{b}, \mathbf{d})$.

By comparing the spectra of cefepime and cefoxitin (Figure 6b,d), we can instead evaluate the chemical sensitivity of optical properties for these molecules, which display several differences in chemical structure although belonging the same cephem family. Despite rather large differences, CD spectra (Figure 6d) of these two molecules display a common negative peak slightly above $180 \mathrm{~nm}$ and more in general they approximately share the pattern of sign variations across the $140-320 \mathrm{~nm}$ region: we can indeed identify a positive region for wavelengths below $180 \mathrm{~nm}$, followed by a negative one between 180 and $200 \mathrm{~nm}$ : for wavelengths larger than $200 \mathrm{~nm}$ the dichroic signal of both molecules lacks strong negative features, although the cefepime spectrum (black line) is positive throughout this region, while the cefoxitin one (red curve) has negligible intensity in the $200 \mathrm{~nm}-260 \mathrm{~nm}$ range, and is slightly negative around $280 \mathrm{~nm}$. On the contrary, the absorption spectra (Figure 6b) of these two cephems differ in number, position, and relative intensity of the few peaks present in the analyzed region.

Interestingly (Figure $7 \mathrm{c}$ ), the $\mathrm{CD}$ spectrum of one of the two studied conformations of the idelalisib molecule (PDB 4XE0, black curve) is quite similar, in the 140-210 nm range, to that of phenylalanine (PHE, orange curve), an amino acid molecule, in one of its two most populated conformers, as calculated by some of us in a previous work [13]. Indeed idelalisib and phenylalanine (Figure 7d) share a phenyl ring, although idelalisib is a larger and more complex molecule with respect to PHE. Moreover, the differences both between the $\mathrm{CD}$ spectra of the two analyzed idelalisib conformers (Figure $7 \mathrm{~b}$ ) and between the $\mathrm{CD}$ spectra of the two most populated PHE conformers (Figure 5B of [13]) are remarkably larger than the difference between the CD spectra of idelalisib and PHE in their conformations shown in Figure 7d. 


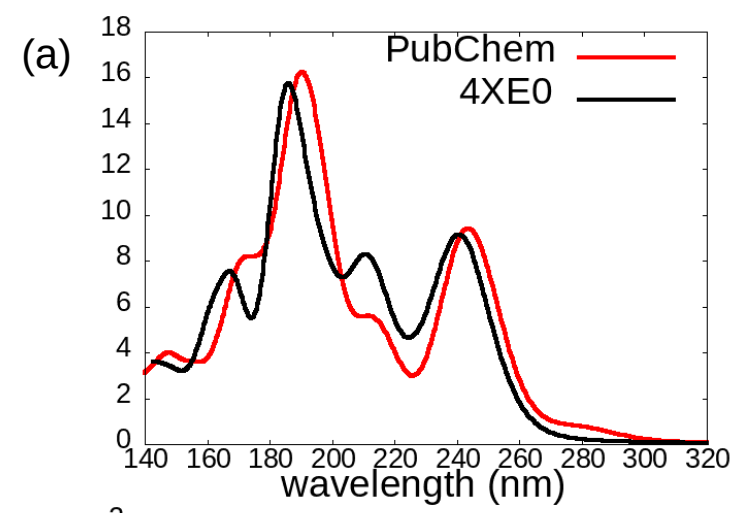

(b)

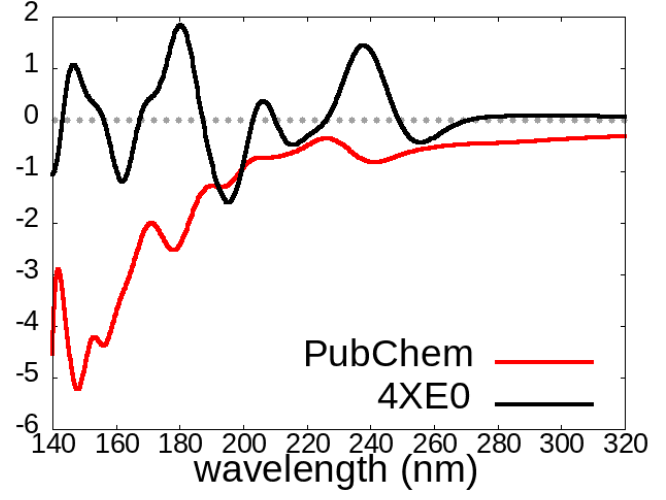

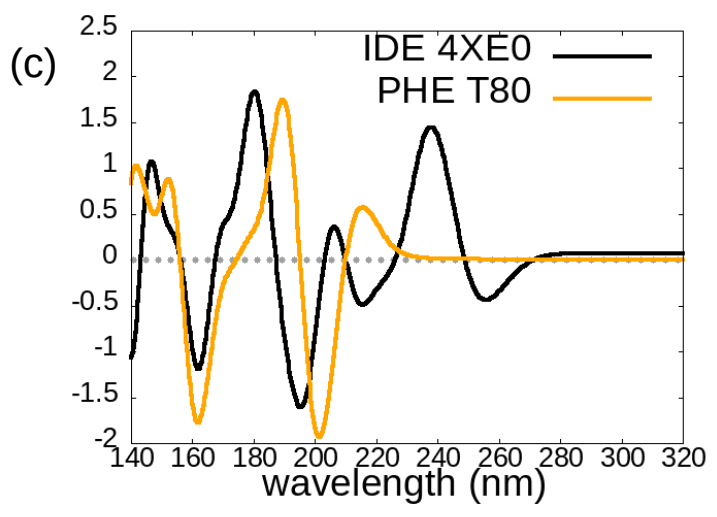

(d)
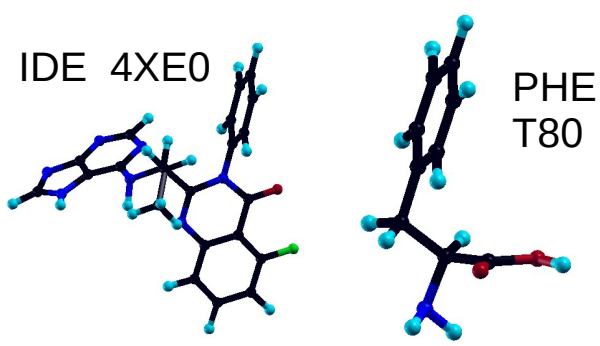

Figure 7. Left panels: comparison of the photoabsorption cross-sections (a) and circular dichroism spectra (b) of the two investigated conformations of the idelalisib molecule. Panel (c) (right): comparison of the circular dichroism spectra of idelalisib 4XE0 conformation and of the phenylalanine (PHE) amino acid in its T80 conformer. The molecular structures for 4XE0-idelalisib and T80-PHE, showing the common phenyl ring, are shown in panel (d).

From the point of view of conformational and chemical sensitivity of absorption and CD spectra, in addition to the ability of CD spectra to identify the absolute configuration (enantiomer), we can thus draw some conclusions.

For the considered conformers of avibactam, both absorption and dichroism spectra are rather sensitive to conformational and chemical details: we cannot identify a "typical" absorption or CD spectrum of avibactam. For the 4ZAM and 4ZBE structures, which share the same chemical structure and protonation (Figure 2 top right) and whose geometric conformations are rather similar, dichroism spectra are negative across the whole investigated spectral region (except for a very weak positive feature around $180 \mathrm{~nm}$ for the $4 Z B E$ one).

For idelalisib, the CD spectrum (Figure $7 \mathrm{~b}$ ) appears to be fundamental in order to distinguish between the two investigated conformers: their absorption spectra (Figure 7a) share indeed all their main features. On the other hand, the circular dichroism spectrum in the 140-210 $\mathrm{nm}$ region appears to be dominated by the signal due to the presence of a phenyl ring, if this latter is in a specific orientation with respect to the other chemical groups: this region may thus be used for identifying the presence of a specific conformer in an idelalisib sample, rather than for distinguishing IDE from different molecular species sharing a phenyl group. Indeed, a marked dependence of chiroptical properties of aromatic amino acids (such as phenylalanine) on their conformation was reported by [33], and our current results may suggest this behavior is shared by a drug molecule like idelalisib, also containing aromatic rings.

For the two considered cephems, overall the absorption spectrum appears to be more diagnostic than the $\mathrm{CD}$ one for distinguishing between cefepime and cefoxitin. However, also the circular dichroism spectrum carries some useful information from this point of view, in that it displays a much more intense negative peak at around $185 \mathrm{~nm}$ for the cefoxitin molecule. 


\section{Materials and Methods}

\subsection{Theory}

Circular dichroism (CD), i.e., the difference in absorption coefficients for right (R) and left (L) circularly polarized light (CPL), can be expressed in terms of the imaginary part of the difference $\Delta n=n_{R}-n_{L}$ between polarization-resolved refraction indexes.

For randomly oriented chiral molecules and within linear response, this difference is proportional to the trace of the $G_{i j}$ tensor [34-37]:

$$
G_{i j}(\omega)=\frac{1}{\hbar} \sum_{n, m}\left(\frac{\left\langle n\left|\hat{\mu}_{i}\right| m\right\rangle\left\langle m\left|\hat{m}_{j}\right| n\right\rangle}{\omega_{n, m}-\omega-i \gamma}+\frac{\left\langle n\left|\hat{m}_{j}\right| m\right\rangle\left\langle m\left|\hat{\mu}_{i}\right| n\right\rangle}{\omega_{n, m}+\omega+i \gamma}\right)
$$

where $\hat{\mu}=q_{e} \hat{\mathbf{r}}$ is the electric dipole operator and $\hat{\mathbf{m}}=\frac{q_{e}}{2 m} \hat{\mathbf{r}} \times \hat{\mathbf{p}}$ is the magnetic dipole one, $|n\rangle$ and $|m\rangle$ are single particle states, $\omega_{n, m}=\omega_{n}-\omega_{m}=\frac{E_{n}-E_{m}}{\hbar}$ is the excitation frequency for an electronic transition between the $|n\rangle$ and $|m\rangle$ states; a finite (and small) value of $\gamma$ is used in order to mimic experimental broadening effects.

By inserting the resolution of the identity, assuming completeness of the basis set, and by taking advantage of the commutator rule involving the $\hat{\mathbf{r}}, \hat{\mathbf{p}}$ and $\hat{H}$ operators (and possibly $V_{n l}\left(r, r^{\prime}\right)$, a nonlocal contribution to the potential), one can choose to evaluate the numerators in Equation (1) in terms of momentum matrix elements only (velocity representation), possibly corrected for the nonlocal contribution.

Thus, the sum of $\left\langle n\left|\hat{\mu}_{i}\right| m\right\rangle\left\langle m\left|\hat{m}_{j}\right| n\right\rangle$ products appearing in the expression of $G_{i j}(\omega)$ turns into a sum of $\left\langle n\left|\hat{p}_{i}\right| m\right\rangle\left\langle m\left|\hat{p}_{j}\right| l\right\rangle\left\langle l\left|\hat{p}_{k}\right| n\right\rangle$ terms. Here the $n$ index runs on valence bands, the $m$ one on conduction bands, and the $l$ one on all bands.

Within this approach, our DFT calculations have been performed within the velocity gauge, using a plane-waves basis set and avoiding any real grid representation of differential operators: this makes computed spectra free from origin-dependence issues, as carefully verified in our previous work on CD spectra of amino acids [13]. Moreover, the choice of a plane-waves basis allows testing numerical convergence in a straightforward way, and makes our circular dichroism code easily interfaceable with existing large-scale, plane-waves DFT codes [38,39].

\subsection{Computational Details}

Self-consistent electronic structure calculations were carried out within density functional theory (DFT) [40,41]: we chose the local density approximation (LDA) exchangecorrelation functional using the Ceperley and Alder results [42] as parametrized by Teter and Pade [43]. We made use of norm-conserving pseudopotentials of the Troullier-Martins type [44], as available on the Abinit [38] website, to treat the ion-electron interaction. KohnSham electronic wave functions and eigenvalues, computed for both occupied and empty states, were used as an input in the calculations of chiroptical spectra.

In order to correct for the expected gap underestimation arising from neglection of self-energy and many-body effects, we applied a scissor operator correction of $1.5 \mathrm{eV}$ to computed absorption and CD spectra, in agreement with the values used in our previous work [13] and validated there by comparison with experimental spectra. Since in the present work the focus is mainly on the chemical and conformational sensitivity of absorption and CD spectra-therefore on the comparison between spectra of different conformers or molecules, rather than on the absolute peak positions-the precise value of the scissor correction is not critical. Calculations were carried out in a cubic supercell of size $20 \times 20 \times 20 \AA$ for all avibactam geometries and for the idealisib 4 XE0 conformer, $24 \times 24 \times 24 \AA$ for the idealisib PubChem conformer, $30 \times 30 \times 30 \AA$ for the two cephems.

\section{Conclusions}

For drug molecules, chirality, i.e., the absence of improper axes of rotation, i.e., planes of symmetry and inversion centers, can have important effects on their efficacy and safety, through stereoselective molecular interactions. Chiroptical properties, due to the different 
interaction of chiral molecules with left or right circularly polarized light, can allow the identification of enantiomers, thus providing information which cannot be accessible through other techniques such as absorption spectroscopy. Circular dichroism is a useful experimental tool in this context, since enantiomers of a chiral molecule yield opposite CD spectra.

Computational investigations can help in several ways, e.g., allowing one to obtain the full CD spectrum of relatively complex molecules, for which predicting the correlation between the sign of the major CD peaks and the absolute configuration based on simple rules may be far from trivial, and highlighting specific spectral regions which are less sensitive to molecule conformation and therefore more diagnostic for its left or right handedness.

In the present paper we have calculated the optical absorption and circular dichroism spectra of some molecules of interest in medical and pharmaceutical research, namely avibactam, as representative of non $\beta$-lactam inhibitors, the two cephems cefepime and cefoxtin, and idelalisib as a recent relevant anticancer active substance used in protocols against major leukemias.

For avibactam, the sensitivity of both absorption and CD spectra to chemical and conformational details is such that one cannot identify a unique "typical" spectrum for this molecule in a fixed absolute configuration (enantiomer). The two chosen conformers of idelalisib are a good example of cases where circular dichroism spectra may provide crucial information-absent in absorption spectra-on the presence of a specific conformer in a sample.

On the other hand, in both the avibactam and idelalisib cases, this strong dependence of CD spectra on chemical and conformational details would hinder their "standard" use for identifying enantiomers.

Moreover, by analyzing the $\mathrm{CD}$ spectrum of a specific conformer of idelalisib, and comparing it with the spectrum of one possible conformer of the phenylalanine amino acid, we have found characteristic CD features, lying in the 140-210 nm range in our computed spectra, which appear to be diagnostic for the presence of a phenyl ring, provided it has a given orientation with respect to the rest of the molecule.

A pronounced negative $C D$ peak such as that we found at $\approx 185 \mathrm{~nm}$ for the chosen enantiomer of cefoxitin may be used either for assigning the absolute configuration of this molecule or to distinguish it from other cephems lacking this spectral feature.

Our results thus allow us to gain some insight on the conformational and chemical sensitivity of the CD spectra of the investigated molecules, also by comparing them with those on other biomolecules previously studied by some of us [13].

These findings contribute to highlighting both the wealth of information contained in circular dichroism spectra of molecules, and the resulting need, in particular for complex molecules containing several chemical groups and often displaying several low energy geometries, for a careful interpretation (possibly supported by computational studies) of $\mathrm{CD}$ measurements for assigning the absolute configuration.

The outcomes of the present research could be of interest within the growing field of studies aiming at exploiting 3D geometry and symmetry properties of drug molecules for improving their pharmacological activity and avoiding adverse effects. Further, their optical identification in solution might be used for assessing accumulation in cells [28].

Possible future work could consist in considering the electronic and optical properties of the above molecules in presence of suitable chemical substitutions $[18,19]$, and interaction effects either with solvent molecules or with biomolecules which may be relevant for their mechanism of activity. In particular, solvent effects on optical properties of either biomolecules of drug molecules deserve attention: as highlighted by some of us in a previous work [13] for the alanine amino acid in water, the presence of solvent can yield several effects: from limiting the experimentally accessible spectral window due to solvent absorption in some spectral regions, to-in the case of polar solvents such as waterfavoring the zwitterionic form of the investigated molecule. Moreover, one should carefully 
choose the computational method(s) to use for those studies, due to nontrivial issues such as spurious circular dichroism contributions arising when using a small (and in general chiral) "cluster" of explicit water molecules for modeling solvation.

Author Contributions: Conceptualization, M.C., G.C.; methodology, E.M., G.O.; software E.M., G.O.; investigation, E.M.; writing—original draft preparation, E.M., G.C.; writing—review and editing, M.C., G.O., G.C.; visualization E.M.; supervision G.C.; funding acquisition, G.C. All authors have read and agreed to the published version of the manuscript.

Funding: This research was funded by Fondazione di Sardegna under Project UniCa "PRID 2015" and by IDEA-AISBL, Bruxelles, Belgium.

Institutional Review Board Statement: Not applicable.

Informed Consent Statement: Not applicable.

Data Availability Statement: All research data are stored according to the Research Data Managenent Policy of Università degli Studi di Milano (rdm@unimi.it), and are available upon request.

Acknowledgments: The authors acknowledge the CINECA award under the ISCRA initiative, for the availability of high-performance computing resources and support (Project HP10CDF3LP).

Conflicts of Interest: The authors declare no conflict of interest.

\section{References}

1. Furman, R.R.; Sharman, J.P.; Coutre, S.E.; Cheson, B.D.; Pagel, J.M.; Hillmen, P.; Barrientos, J.C.; Zelenetz, A.D.; Kipps, T.J.; Flinn, I.; et al. Idelalisib and Rituximab in Relapsed Chronic Lymphocytic Leukemia. N. Engl. J. Med. 2014, 370, 997-1007. [CrossRef] [PubMed]

2. Vergalli, J.; Bodrenko, I.V.; Masi, M.; Moynié, L.; Acosta-Gutierrez, S.; Naismith, J.H.; Davin-Régli, A.; Ceccarelli, M.; van den Berg, B.; Winterhalter, M.; et al. Porins and small-molecule translocation across the outer membrane of Gram-negative bacteria. Nat. Rev. Microbiol. 2020, 18, 164-176. [CrossRef] [PubMed]

3. Qin, W.; Panunzio, M.; Biondi, S. $\beta$-Lactam Antibiotics Renaissance. Antibiotics 2014, 3, 193-215. [CrossRef] [PubMed]

4. Ehmann, D.E.; Jahić, H.; Ross, P.L.; Gu, R.F.; Hu, J.; Kern, G.; Walkup, G.K.; Fisher, S.L. Avibactam is a covalent, reversible, non- $\beta$-lactam $\beta$-lactamase inhibitor. Proc. Natl. Acad. Sci. USA 2012, 109, 11663-11668. [CrossRef]

5. Wang, D.Y.; Abboud, M.I.; Markoulides, M.S.; Brem, J.; Schofield, C.J. The road to avibactam: The first clinically useful non- $\beta$-lactam working somewhat like a $\beta$-lactam. Future Med. Chem. 2016, 8, 1061. [CrossRef]

6. Carmeli, Y.; Armstrong, J.; Laud, P.J.; Newell, P.; Stone, G.; Wardman, A.; Gasink, L.B. Ceftazidime-avibactam or best available therapy in patients with ceftazidime-resistant Enterobacteriaceae and Pseudomonas aeruginosa complicated urinary tract infections or complicated intra-abdominal infections (REPRISE): A randomised, pathogen-directed, phase 3 study. Lancet Infect. Dis. 2016, 16, 661-673. [CrossRef]

7. Abboud, M.I.; Damblon, C.; Brem, J.; Smargiasso, N.; Mercuri, P.; Gilbert, B.; Rydzik, A.M.; Claridge, T.D.W.; Schofield, C.J.; Frère, J.M. Interaction of Avibactam with Class B Metallo- $\beta$-lactamases. Antimicrob. Agents Chemother. 2016, 60, 5655-5662. [CrossRef]

8. Ehmann, D.; Jahić, H.; Ross, P.L.; Gu, R.F.; Hu, J.; Durand-Réville, T.F.; Lahiri, S.; Thresher, J.; Livchak, S.; Gao, N.; et al. Kinetics of Avibactam Inhibition against Class A, C, and D $\beta$-Lactamases. J. Biol. Chem. 2013, 288, 27960-27971. [CrossRef]

9. Pira, A.; Scorciapino, M.A.; Bodrenko, I.V.; Bosin, A.; Acosta-Gutierrez, S.; Ceccarelli, M. Permeation of $\beta$-Lactamase Inhibitors through the General Porins of Gram-Negative Bacteria. Molecules 2020, 25, 5747. [CrossRef]

10. Okamoto, M.P.; Nakahiro, R.K.; Chin, A.; Bedikian, A.; Gill, M.A. Cefepime: A new fourth-generation cephalosporin. Am. J. Hosp. Pharm. 1994, 51, 463-477. [CrossRef]

11. Birnbaum, J.; Stapley, E.O.; Miller, A.K.; Celozzi, E.; Wallick, H.; Pelak, B.A.; Zimmerman, S.B.; Hendlin, D.; Woodruff, H.B. Development of the semisynthetic cephamycin, cefoxitin, as a clinical candidate. Infection 1979, 7, S13-S20. [CrossRef]

12. Saha, D.; Kharbanda, A.; Yan, W.; Lakkaniga, N.R.; Frett, B.; Li, H.Y. The Exploration of Chirality for Improved Druggability within the Human Kinome. J. Med. Chem. 2020, 63, 441-469. [CrossRef]

13. Molteni, E.; Onida, G.; Tiana, G. Conformational Dependence of the Circular Dichroism Spectra of Single Amino Acids from Plane-Waves-Based Density Functional Theory Calculations. J. Phys. Chem. B 2015, 119, 4803-4811. [CrossRef]

14. Gortàzar, P.; Vàzquez, J.T. Discrimination and Direct Determination of Cephalosporins by Circular Dichroism. J. Pharm. Sci. 1994, 83, 1204-1208. [CrossRef]

15. Berova, N.; Bari, L.D.; Pescitelli, G. Application of electronic circular dichroism in configurational and conformational analysis of organic compounds. Chem. Soc. Rev. 2007, 36, 914-931. [CrossRef]

16. Ceccarelli, M.; Danelon, C.; Laio, A.; Parrinello, M. Microscopic Mechanism of Antibiotics Translocation through a Porin. Biophys. J. 2004, 87, 58-64. [CrossRef]

17. Ceccarelli, M.; Vargiu, A.V.; Ruggerone, P. A kinetic Monte Carlo approach to investigate antibiotic translocation through bacterial porins. J. Phys. Condens. Matter 2012, 24, 104012. [CrossRef] 
18. Molteni, E.; Onida, G.; Cappellini, G. Electronic structure of uracil-like nucleobases adsorbed on Si(001): uracil, thymine and 5-fluorouracil. Eur. Phys. J. B 2016, 89, 98. [CrossRef]

19. Molteni, E.; Cappellini, G.; Onida, G.; Fratesi, G. Optical Properties of Organically Functionalized Silicon Surfaces: Uracil-like Nucleobases on Si(001). Phys. Rev. B 2017, 95, 075437. [CrossRef]

20. Cardia, R.; Cappellini, G.; Pinna, E.; Tiddia, M.; Mula, G. Optical and electronic properties of monomers of eumelanin: A DFT and TD-DFT computational study. Opt. Photonics J. 2016, 6, 41-47. [CrossRef]

21. Molteni, E.; Cappellini, G.; Onida, G.; Mula, G. Extensive Stacking of DHI-like Monomers as a Model of Out-of-Plane Complexity in Eumelanin Protomolecules: Chemical and Structural Sensitivity of Optical Absorption Spectra. Chem. Phys. 2019, 524, 92-100. [CrossRef]

22. Molteni, E.; Cappellini, G.; Cardia, R.; Onida, G.; Mula, G. Eumelanin Adsorption on Silicon: Optical Properties of Si(001)Adsorbed Eumelanin Tetrameric Protomolecules. J. Phys. Chem. C 2020, 124, 9376-9384. [CrossRef]

23. Pinna, E.; Melis, C.; Antidormi, A.; Cardia, R.; Sechi, E.; Cappellini, G.; d'Ischia, M.; Colombo, L.; Mula, G. Deciphering Molecular Mechanisms of Interface Buildup and Stability in Porous Si/Eumelanin Hybrids. Int. J. Mol. Sci. 2017, 18, 1567. [CrossRef]

24. Antidormi, A.; Aprile, G.; Cappellini, G.; Cara, E.; Cardia, R.; Colombo, L.; Farris, R.; d'Ischia, M.; Mehrabanian, M.; Melis, C.; et al. Physical and Chemical Control of Interface Stability in Porous Si-Eumelanin Hybrids. J. Phys. Chem. C 2018, 122, $28405-28415$. [CrossRef]

25. Kumar, A.; Cardia, R.; Cappellini, G. Electronic and Optical Properties of Chromophores from Bacterial Cellulose. Cellulose 2018, 25, 2191-2203. [CrossRef]

26. Kumar, A.; Cappellini, G.; Delogu, F. Electronic and Optical Properties of Chromophores from Hexeneuronic Acids. Cellulose 2019, 26, 1489-1501. [CrossRef]

27. Kascàkovà, S.; Maigre, L.; Chevalier, J.; Réfrégiers, M.; Pagès, J.M. Antibiotic Transport in Resistant Bacteria: Synchrotron UV Fluorescence Microscopy to Determine Antibiotic Accumulation with Single Cell Resolution. PLoS ONE 2012, 7, e38624-9. [CrossRef]

28. Prochnow, H.; Fetz, V.; Hotop, S.K.; García-Rivera, M.A.; Heumann, A.; Brönstrup, M. Subcellular Quantification of Uptake in Gram-Negative Bacteria. Anal. Chem. 2018, 91, 1863-1872. [CrossRef]

29. Lahiri, S.D.; Mangani, S.; Jahić, H.; Benvenuti, M.; Durand-Reville, T.F.; Luca, F.D.; Ehmann, D.E.; Rossolini, G.M.; Alm, R.A.; Docquie, J.D. Molecular Basis of Selective Inhibition and Slow Reversibility of Avibactam against Class D Carbapenemases: A Structure-Guided Study of OXA-24 and OXA-48. ACS Chem. Biol. 2015, 10, 591-600. [CrossRef]

30. Krishnan, N.P.; Nguyen, N.Q.; Papp-Wallace, K.M.; Bonomo, R.A.; van den Akker, F. Inhibition of Klebsiella $\beta$-Lactamases (SHV-1 and KPC-2) by Avibactam: A Structural Study. PLoS ONE 2015, 10, e0136813. [CrossRef]

31. Somoza, J.R.; Koditek, D.; Villaseñor, A.G.; Novikov, N.; Wong, M.H.; Liclican, A.; Xing, W.; Lagpacan, L.; Wang, R.; Schultz, B.E.; et al. Structural, Biochemical, and Biophysical Characterization of Idelalisib Binding to Phosphoinositide 3-Kinase

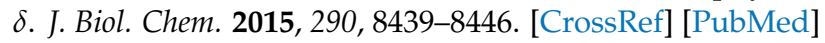

32. Malloci, G.; Vargiu, A.V.; Serra, G.; Bosin, A.; Ruggerone, P.; Ceccarelli, M. A Database of Force-Field Parameters, Dynamics, and Properties of Antimicrobial Compounds. Molecules 2015, 20, 13997-14021. [CrossRef] [PubMed]

33. Kundrat, M.D.; Autschbach, J. Time Dependent Density Functional Theory Modeling of Chiroptical Properties of Small Amino Acids in Solution. J. Phys. Chem. A 2006, 110, 12908-12917. [CrossRef] [PubMed]

34. Rosenfeld, L. Quantenmechanische Theorie der Natürlichen Optischen Aktivität von Flüssigkeiten und Gasen. Z. Phys. 1929, 52, 161-174. [CrossRef]

35. Caldwell, D.J. The Theory of Optical Activity; Wiley-Interscience: New York, NY, USA, 1971.

36. Condon, E.U. Theories of Optical Rotatory Power. Rev. Mod. Phys. 1937, 9, 432-457. [CrossRef]

37. Barron, L. Molecular Light Scattering and Optical Activity; Cambridge University Press: Cambridge, UK, 2004.

38. Gonze, X.; Amadon, B.; Anglade, P.M.; Beuken, J.M.; Bottin, F.; Boulanger, P.; Bruneval, F.; Caliste, D.; Caracas, R.; Cote, M.; et al. ABINIT: First-Principles Approach to Material and Nanosystem Properties. Comput. Phys. Commun. 2009, 180, $2582-2615$. [CrossRef]

39. Giannozzi, P.; Andreussi, O.; Brumme, T.; Bunau, O.; Nardelli, M.B.; Calandra, M.; Car, R.; Cavazzoni, C.; Ceresoli, D.; Cococcioni, M.; et al. Advanced Capabilities for Materials Modelling with Quantum ESPRESSO. J. Phys. Condens. Matter 2017, 29, 465901. [CrossRef]

40. Hohenberg, P.; Kohn, W. Inhomogeneous Electron Gas. Phys. Rev. 1964, 136, B864. doi:10.1103/PhysRev. 136.B864. [CrossRef]

41. Kohn, W.; Sham, L.J. Self-Consistent Equations Including Exchange and Correlation Effects. Phys. Rev. 1965, 140, A1133-A1138. [CrossRef]

42. Ceperley, D.M.; Alder, B.J. Ground State of the Electron Gas by a Stochastic Method. Phys. Rev. Lett. 1980, 845, 566. [CrossRef]

43. Goedecker, S.; Teter, M.; Huetter, J. Separable Dual-space Gaussian Pseudopotentials. Phys. Rev. B 1996, 54, 1703. [CrossRef]

44. Troullier, N.; Martins, J.L. Efficient Pseudopotentials for Plane-Wave Calculations. Phys. Rev. B 1991, 43, 1993. [CrossRef] 